\title{
Prevenção e tratamento da lesão por pressão na atualidade: revisão de literatura
}

\author{
Prevention and treatment of pressure injuries today: literature review \\ Prevención y tratamiento de las lesiones por presión en la actualidad: revisión de la literatura
}

\author{
Giovanna da Silva Soares Souza \\ ORCID: https://orcid.org/0000-0002-4861-5659 \\ Centro Universitário Facid Wyden, Brasil \\ E-mail: giovanna40444@hotmail.com \\ Laurice Alves dos Santos \\ ORCID: https://orcid.org/0000-0003-0362-3257 \\ Centro Universitário Facid Wyden, Brasil \\ E-mail: lauricealvesenf@hotmail.com \\ Alessandro Monteiro Carvalho \\ ORCID: https://orcid.org/0000-0001-8257-6916 \\ Universidade Federal do Piauí, Brasil \\ E-mail: alefarma443@yahoo.com.br \\ Pedrina Maria Nascimento Araújo Costa \\ ORCID: https://orcid.org/0000-0002-4208-9168 \\ Centro Universitário Santo Agostinho, Brasil \\ E-mail: pedrinaaraujo-fartura@hotmail.com \\ Taniel Lopes da Silva \\ ORCID: https://orcid.org/0000-0001-6759-3363 \\ Centro Universitário Maurício de Nassau, Brasil \\ E-mail: taniel.1s@hotmail.com
}

\begin{abstract}
Resumo
Objetivo: Identificar e analisar sobre as formas de prevenção e tratamento da LPP na atualidade. Método: Trata-se de uma revisão integrativa da literatura, com uma abordagem quantitativa, feita através de levantamentos bibliográficos, utilizando, periódicos indexados em bases de dados nacionais (SCIELO, PORTAL BVS, PUBMED E LILACS, CAPES PERIÓDICOS). Resultados: Os resultados evidenciam que as formas mais eficientes de profilaxia é uma boa inspeção da pele, mudança de decúbito, e o uso de tratamento tópico sistêmico das lesões por pressão, que irá considerar os procedimentos de limpeza, uso de coberturas primárias e secundárias ou de fixação. Conclusão: Por meio deste estudo proposto espera-se a investigação das lesões por pressão assim como as profilaxias e tratamento adequado.
\end{abstract}

Palavras-chave: Lesão por pressão; Profilaxias; Tratamento.

\begin{abstract}
Objective: To identify and analyze forms of prevention and treatment of LPP today. Method: This is an integrative literature review, with a quantitative approach, carried out through bibliographic surveys, using journals indexed in national databases (SCIELO, PORTAL BVS, PUBMED AND LILACS, CAPES PERIODICOS). Results: The results show that the most efficient forms of prophylaxis are a good inspection of the skin, change of position, and the use of systemic topical treatment of pressure injuries, which will consider cleaning procedures, use of primary and secondary dressings or of attachment. Conclusion: Through this proposed study, investigation of pressure injuries is expected, as well as prophylaxis and appropriate treatment.
\end{abstract}

Keywords: Pressure injury; Prophylaxis; Treatment.

\section{Resumen}

Objetivo: Identificar y analizar formas de prevención y tratamiento de la LPP en la actualidad. Método: Se trata de una revisión integradora de la literatura, con enfoque cuantitativo, realizada a través de encuestas bibliográficas, utilizando revistas indexadas en bases de datos nacionales (SCIELO, PORTAL BVS, PUBMED Y LILACS, CAPES PERIODICOS). Resultados: Los resultados muestran que las formas más eficientes de profilaxis son una buena inspección de la piel, el cambio de posición y el uso de tratamiento tópico sistémico de las lesiones por presión, que considerarán procedimientos de limpieza, uso de apósitos primarios y secundarios o de fijación. Conclusión: A través de este estudio propuesto, se espera la investigación de las lesiones por presión, así como la profilaxis y el tratamiento adecuado.

Palabras clave: Lesión por presión; Profilaxis; Tratamiento. 


\section{Introdução}

As lesões por pressão podem ser identificadas como qualquer lesão ocasionada por uma pressão não aliviada, cisalhamento ou fricção, que podem resultar em morte tecidual. Sendo regularmente localizada na região de prominências ósseas. Sendo classificadas em quatro estágios importantes, para o melhor diagnóstico da lesão. (Alencar et al., 2018).

O estágio 1 possui uma pele íntegra com eritema que não embranquece, estágio 2 é a perda da pele em sua espessura parcial com exposição da derme, estágio 3 é a perda da pele em sua espessura total, estágio 4 é a perda da pele em sua espessura total e perda tissular. As lesões por pressão ainda podem ser classificadas como lesão por pressão não classificável é a perda da espessura dos tecidos, na qual a base da úlcera está coberta por tecido desvitalizado (amarelo, necrótico, cinzentos) no leito da ferida, a lesão por pressão tissular profunda é uma área vermelha escura ou púrpura localizada em pele intacta e deslocada ou flitena preenchida com sangue, provocado por danos no tecido mole subjacente resultantes de pressão e/ou cisalhamento. (Guideline,2019).

Portanto, destacam-se como as principais e mais eficientes formas de prevenção de LPP: inspeção da pele, mudança de decúbito de 2 em 2 horas, uso de colchões especiais, controle de peso, hidratação e como prevenção ao uso de hidrocoloides que proporcionam uma absorção maior da umidade, auxiliando na cicatrização da lesão, entre outros. (Asari et al., 2014).

O tratamento de lesão por pressão deverá ser implementado em conjunto com as medidas preventivas. Irão considerar os tratamentos de LPP, a terapia tópica adotada, cujo são realizados procedimentos de limpeza e de desbridamento, o uso de (coberturas primárias, secundárias e de fixação) e as intervenções sistêmicas voltadas para o controle/ajustes de fatores intrínsecos, que interfere diretamente ou indiretamente no processo de cicatrização da LPP (Santos et al., 2016).

Ainda, a partir do viés investigado acerca da literatura, temos que para identificar possíveis lesões a equipe de enfermagem utiliza o método da Escala de Braden indicada pela Agência Nacional de Vigilância Sanitária (ANVISA), que tem por objetivo avaliar seis principais características do paciente: percepção sensorial, umidade, atividade, mobilidade, nutrição, fricção e cisalhamento. Onde são pontuadas de 1 a 4 sendo que quanto melhor o estado do paciente, maior será a pontuação, depois de feito a somatória total das características pontuação varia entre 6 e 23, e a partir desses dados o enfermeiro classifica o risco do paciente em relação a desenvolver a LPP, onde quanto menor os pontos desta somatória maior o risco (Oliveira G.S.M, Silva et al., 2021).

As lesões da pele necessitam de atenção especial por parte dos profissionais de saúde, destacando-se o papel do enfermeiro, que busca novos conhecimentos para fundamentar sua prática (Favreto, et al., 2017).

Neste contexto, a equipe de Enfermagem tem grande importância na prevenção e tratamento das LPP, pois tem uma atuação de forma direta e contínua ao indivíduo doente. Ao mesmo tempo sabe-se que estes profissionais em muitas instituições, públicas e privadas, trabalham com insuficiência de materiais e equipamentos para tal, assim como, vivenciam uma sobrecarga de trabalho dificultando assim, uma assistência segura e com qualidade (Freitas, 2013, Pachá, et al., 2018).)

Este estudo propõe identificar e analisar as medidas de prevenção e tratamento da LPP, com a formulação da seguinte questão de pesquisa: " Quais as formas de prevenção e tratamento da LPP na atualidade"”? o intuito de atingir o objeto desse estudo, foram elaborados os seguintes objetivos: Averiguar as estratégias de prevenção e dos fatores de risco causadores de lesão por pressão, medidas adotadas nos casos de lesão por pressão. A relevância desse estudo, através de uma revisão de literatura, é enfatizar sobre as profilaxias da lesão por pressão, assim como as formas de tratamentos atuais das lesões. Mostrando as etapas de cada estágio e a melhor conduta para com o paciente. Logo, que é de grande importância o conhecimento dos profissionais de enfermagem para uma melhor abordagem com o paciente. 


\section{Metodologia}

O presente estudo trata-se de uma revisão integrativa de literatura através de uma pesquisa. Com o intuito de sumarizar e revisar as principais evidências cientificas publicadas e obter conclusões a partir da pergunta norteadora sobre a prevenção e tratamento de lesão por pressão.

O levantamento bibliográfico foi realizado na busca de dados científicos por meio de indexadores online: SCIELO, PORTAL BVS, PUBMED E LILACS, CAPES PERIÓDICOS. A busca ocorreu no período de agosto a novembro de 2021. Utilizaram-se os seguintes descritores: lesão por pressão, profilaxias, tratamento.

Foram incluídas revisões de literatura, monografias, dissertações, teses. Os quais apresentaram disponibilidade de texto completo, em idiomas português, inglês, que apresentavam coerência com a temática, publicados nos últimos 10 anos, compreendendo o período entre 2012 e 2021. Foram excluídas, publicações cujos títulos e/ou objetivos não possuíam ligação direta com a temática ou que fugiam do objeto de estudo.

A coleta de dados se baseou em um levantamento sistemático do material, o qual foi obtido por meio de downloads dos arquivos disponíveis em PDF. Os dados coletados obedeceram aos critérios de inclusão, objetivos propostos na pesquisa e temática em estudo. Em seguida, foi feita uma análise das pesquisas a fim de verificar quais artigos corroboravam ou discordavam do assunto. Os resultados foram organizados em forma de quadros, da qual o Quadro 1, aponta as informações em cada obra e os seus dados relevantes título, objetivo, métodos, ano, e autores a respeito da temática, no Quadro 2, identifica a utilização da escala de braden como prevenção de LPP, no Quadro 3, mostra os escores

\section{Resultados e Discussão}

A seguir será elencada em quadros os resultados obtidos na revisão integrativa, as informações estão elencadas abaixo no Quadro 1, com as devidas informações como título, ano, metodologia e conclusão de artigos que foram selecionados para concluir a pesquisa deste trabalho, Quadro 2, identifica a utilização da escala de braden e o Quadro 3 os escores para identificar a escala de braden.

Quadro 1. Apresentação dos artigos incluídos na revisão integrativa.

\begin{tabular}{|c|c|c|c|}
\hline TÍTULO & ANO & MÉTODO & CONCLUSÃO \\
\hline $\begin{array}{l}\text { Lesão por pressão na } \\
\text { unidade de terapia } \\
\text { intensiva: incidência } \\
\text { de fatores de riscos } \\
\end{array}$ & 2018 & $\begin{array}{l}\text { Trata-se de uma revisão integrativa de literatura. A } \\
\text { seleção da amostragem foi realizada a partir dos bancos } \\
\text { de dados SCIELO, LILACS e BDENF, sendo } \\
\text { selecionados estes artigos. }\end{array}$ & $\begin{array}{l}\text { De acordo com as publicações analisadas, infere-se que as } \\
\text { LPP são agravos presentes em unidades hospitalares que } \\
\text { atendem a pacientes críticos devido á restrição permanente ou } \\
\text { temporária de motilidade presente em muitos dos casos. }\end{array}$ \\
\hline $\begin{array}{l}\text { O papel do enfermeiro } \\
\text { na prevenção, } \\
\text { avaliação e tratamento } \\
\text { das lesões por pressão }\end{array}$ & 2017 & $\begin{array}{l}\text { Foram utilizados artigos dos últimos } 12 \text { anos que } \\
\text { abordam assuntos relacionados diretamente as lesões por } \\
\text { pressão }\end{array}$ & $\begin{array}{l}\text { Conclui-se que o enfermeiro tem o papel fundamental na } \\
\text { prevenção, avaliação e tratamento dos pacientes portadores } \\
\text { de lesão por pressão, treinando a equipe com relação as novas } \\
\text { técnicas resultando no melhor tratamento possível }\end{array}$ \\
\hline $\begin{array}{c}\text { Prevenção e } \\
\text { tratamento de lesão } \\
\text { por pressão }\end{array}$ & 2020 & $\begin{array}{l}\text { Foi feito a revisão do documento e adequada ao novo } \\
\text { modelo }\end{array}$ & \\
\hline $\begin{array}{l}\text { Lesão por pressão } \\
\text { relacionada a } \\
\text { dispositivos médicos: } \\
\text { revisão integrativa de } \\
\text { literatura }\end{array}$ & 2019 & $\begin{array}{l}\text { Revisão integrativa, com busca nas bases: CINAHL, } \\
\text { PubMed, Wiley interscience, Scopus e Web of Science. } \\
\text { Utilizou-se, os termos "pressure ulcer" e "medical } \\
\text { devices" sendo incluídos artigos originais e estudos de } \\
\text { casos, publicados entre } 2010 \text { e } 2015 \text {. Foram selecionados } \\
\text { nove estudos }\end{array}$ & $\begin{array}{l}\text { As lesões por pressão relacionados a dispositivos médicos são } \\
\text { problemas frequentes, todavia, podem ser prevenidas e } \\
\text { tratadas com base nas recomendações específicas ao } \\
\text { dispositivo empregado e medidas gerais }\end{array}$ \\
\hline $\begin{array}{l}\text { Classificação das } \\
\text { lesões por pressão } \\
\text { adaptado } \\
\text { culturalmente para o } \\
\text { brasil }\end{array}$ & 2016 & ---------- & --------------- \\
\hline
\end{tabular}


Research, Society and Development, v. 10, n. 17, e61101723945, 2021

\begin{tabular}{|c|c|c|c|}
\hline $\begin{array}{l}\text { Lesão por pressão: } \\
\text { medidas terapêuticas } \\
\text { utilizadas por } \\
\text { profissionais de } \\
\text { enfermagem }\end{array}$ & 2019 & $\begin{array}{l}\text { Estudo descritivo exploratório com abordagem } \\
\text { quantitativa, realizado nas clínicas: Médica, Cirúrgica e } \\
\text { UTI de hospital de ensino em João Pessoa/PB. Realizado } \\
\text { com a equipe de enfermagem por meio de um } \\
\text { questionário estruturado com variáveis de caracterização } \\
\text { dos participantes e de cuidados preventivos e terapia } \\
\text { tópica da LPP. }\end{array}$ & $\begin{array}{l}\text { Os principais resultados apontam para uma conformidade } \\
\text { entre, as ações e recomendações da literatura, no entanto } \\
\text { acredita-se que é necessário investir em educação permanente } \\
\text { sobre atualidades em coberturas/curativos, para que os } \\
\text { profissionais de enfermagem tenham um embasamento } \\
\text { cientifico mais significativo que permita atuar com segurança }\end{array}$ \\
\hline $\begin{array}{l}\text { Cuidados de } \\
\text { enfermagem na } \\
\text { prevenção de lesão por } \\
\text { pressão }\end{array}$ & 2016 & $\begin{array}{l}\text { Trata se de uma revisão bibliográfica na qual foram } \\
\text { analisados artigos encontrados nos sites da Scielo, Web } \\
\text { of Science, Science Direct e livros publicados de } 2000 \text { a } \\
2016\end{array}$ & $\begin{array}{l}\text { Conclui-se que a prevenção das lesões por pressão estấ } \\
\text { diretamente ligada aos cuidados da enfermagem, dispondo de } \\
\text { medidas simples, porém eficazes. }\end{array}$ \\
\hline $\begin{array}{l}\text { Prevenção e } \\
\text { tratamento de úlceras } \\
\text { por pressão: guia de } \\
\text { consulta rápida. }\end{array}$ & 2016 & $\begin{array}{l}\text { Foi utilizada uma metodologia científica específica para } \\
\text { identificar e avaliar de forma crítica a investigação } \\
\text { disponível. Na ausência de evidência definitiva, } \\
\text { recorreu-se à opinião de peritos (muitas vezes } \\
\text { fundamentada em evidência indireta e outras diretrizes) } \\
\text { com vista à formulação de recomendações. As versões } \\
\text { preliminares das recomendações e a evidência disponível } \\
\text { foram disponibilizadas a } 986 \text { stakeholders convidados } \\
\text { (pessoas individuais e organizações) em todo o mundo. } \\
\text { A versão final do Guia baseia-se na investigação } \\
\text { existente e na sabedoria acumulada do NPUAP, do } \\
\text { EPUAP, da PPPIA e de parceiros internacionais. }\end{array}$ & $\begin{array}{l}\text { Indica também ao profissional de saúde o grau de confiança } \\
\text { que poderá ter na recomendação para saber se esta será mais } \\
\text { benéfica do que prejudicial. Pode ainda ser utilizada para } \\
\text { auxiliar na priorização de intervenções relacionadas com } \\
\text { úlceras por pressão. }\end{array}$ \\
\hline $\begin{array}{l}\text { Ensino de prevenção e } \\
\text { tratamento de lesão } \\
\text { por pressão utilizando } \\
\text { simulação }\end{array}$ & 2018 & $\begin{array}{l}\text { Relato de experiência de construção de cenário clinico } \\
\text { simulado de alta fidelidade, com base na literatura e } \\
\text { Taxonomia de Bloom. Foram considerados objetivos de } \\
\text { aprendizagem, material de estudo prévio, fidelidade, } \\
\text { caso clínico, recursos necessários, resolução de } \\
\text { problemas, validação de aparência e conteúdo, } \\
\text { debriefing, avaliação. }\end{array}$ & $\begin{array}{l}\text { A construção de cenários clínicos simulados planejados, } \\
\text { estruturados e validados, aproxima o aprendiz da prática } \\
\text { clínica possibilitando o desenvolvimento de competências } \\
\text { necessárias para a prevenção e tratamento de lesão por } \\
\text { pressão. Implicações para a prática: em cenários clínicos } \\
\text { simulados bem delineados aproximam com fidelidade o } \\
\text { treino da assistência de enfermagem ao paciente com lesão } \\
\text { por pressão. }\end{array}$ \\
\hline $\begin{array}{l}\text { Construção e } \\
\text { validação de algoritmo } \\
\text { para tratamento da } \\
\text { lesão por pressão }\end{array}$ & 2017 & $\begin{array}{l}\text { Estudo quantitativo, prospectivo, descritivo, analítico } \\
\text { para a elaboração do algoritmo; realizou-se ampla } \\
\text { revisão da literatura. Trinta e oito enfermeiros } \\
\text { especialistas participaram na validação do algoritmo. }\end{array}$ & $\begin{array}{l}\text { O algoritmo na versão validada mostrou confiabilidade para } \\
\text { tratamento da lesão de pressão. }\end{array}$ \\
\hline $\begin{array}{l}\text { Lesão por pressão: } \\
\text { uma revisão de } \\
\text { literatura }\end{array}$ & 2018 & $\begin{array}{l}\text { Utilizou-se uma pesquisa bibliográfica com abordagem } \\
\text { qualitativa descritiva, fundamentada em produções } \\
\text { científicas disponíveis em base de dados indexadas } \\
\text { (PubMed, Lilacs e SciELO, na língua inglesa e } \\
\text { portuguesa). Foram consultadas 35 referências } \\
\text { publicadas entre } 1987 \text { e 2017. Observou-se que há vários } \\
\text { dados presentes na literatura sobre LP, mas a maioria } \\
\text { aborda temas específicos. }\end{array}$ & $\begin{array}{l}\text { Assim, essa revisão possibilitou uma maior compreensão das } \\
\text { principais características das LP, bem como dos } \\
\text { procedimentos a serem adotados durante o atendimento aos } \\
\text { pacientes com essas feridas, visando sempre o melhor } \\
\text { atendimento e o melhor tratamento possível }\end{array}$ \\
\hline $\begin{array}{l}\text { Curativos de lesões } \\
\text { por pressão em } \\
\text { pacientes críticos: } \\
\text { análise de custos }\end{array}$ & 201 & $\begin{array}{l}\text { Os dados foram coletados por meio doEscala de Úlcera } \\
\text { por Pressão para Cura e formulário para caracterização e } \\
\text { avaliação de custos. Valores emReais brasileiros (BRL) } \\
\text { foram convertidos em dólares americanos à taxa de } \\
\text { câmbio de US } \$ 0,26 / \text { BRL. Análises univariadas e } \\
\text { bivariadas foram conduzidas. }\end{array}$ & $\begin{array}{l}\text { A redução dos custos foi proporcional ao estágio da úlcera } \\
\text { por pressão. oO papel dos enfermeiros na criação de planos } \\
\text { de cuidados baseados em evidências é crucial para melhorar } \\
\text { a gestão do cuidado }\end{array}$ \\
\hline $\begin{array}{l}\text { Escala de Braden: } \\
\text { benefícios de sua } \\
\text { aplicação na } \\
\text { prevenção de lesão por } \\
\text { pressão no âmbito } \\
\text { domiciliar }\end{array}$ & 2021 & $\begin{array}{l}\text { Trata-se de um estudo descritivo com abordagem } \\
\text { quantitativa com coleta de dados realizada em domicílio } \\
\text { por meio de entrevista e inspeção da pele de } 41 \\
\text { participantes }\end{array}$ & $\begin{array}{l}\text { Apesar de ser um instrumento bastante utilizado pelos } \\
\text { profissionais da área da saúde, o presente trabalho } \\
\text { demonstrou que a alta sensibilidade e especificidade da } \\
\text { Escala de Braden é questionável. Os cuidados foram } \\
\text { essênciais para essa baixa incidência de lesão por pressão, } \\
\text { diminuindo sua associação com a idade e tempo de acamado }\end{array}$ \\
\hline $\begin{array}{l}\text { Prevenção e } \\
\text { tratamento de } \\
\text { úlceras/lesões por } \\
\text { pressão: guia de } \\
\text { consulta rápida } 2019\end{array}$ & 2019 & $\begin{array}{l}\text { A metodologia foi pré-publicada e revisada por pares. } \\
\text { Uma pesquisa atualizada da literatura identificou } \\
\text { pesquisas publicadas até agosto de } 2018 \text { que foram } \\
\text { avaliadas e analisadas criticamente. Novas pesquisas } \\
\text { foram combinadas com pesquisas de edições anteriores } \\
\text { para ampliar o escopo das diretrizes e produzir } \\
\text { recomendações que refletem as evidências mais recentes. } \\
\text { Esta terceira edição fornece } 115 \text { recomendações } \\
\text { baseadas em evidências, suportadas por uma visão geral } \\
\text { da pesquisa subjacente. }\end{array}$ & $\begin{array}{l}\text { O Guideline Internacional de Prática Clínica (edição 2019) } \\
\text { O Guideline de Prática Clínica fornece uma análise mais } \\
\text { abrangente e detalhada das evidências apoiadas em } \\
\text { recomendações e declarações de boas práticas e inclui } \\
\text { considerações importantes de implementação que fornecem } \\
\text { um contexto adicional às declarações incluídas no Guia de } \\
\text { Consulta Rápida. Este Guia de Consulta Rápida é destinado a } \\
\text { profissionais de saúde ocupados que precisam de uma } \\
\text { referência rápida no cenário clínico }\end{array}$ \\
\hline $\begin{array}{l}\text { A Escala de Braden na } \\
\text { avaliação do risco para } \\
\text { lesão por pressão }\end{array}$ & 2020 & $\begin{array}{l}\text { Estudo transversal, quantitativo, que avaliou, com a } \\
\text { Escala de Braden, pacientes internados em uma UTI } \\
\text { entre novembro de } 2016 \text { e fevereiro de } 2017\end{array}$ & $\begin{array}{l}\text { O desempenho da Escala de Braden apresentou equilíbrio } \\
\text { entre sensibilidade e especificidade, mostrando-se melhor } \\
\text { instrumento preditivo de risco nessa clientela. }\end{array}$ \\
\hline $\begin{array}{l}\text { Uso de escala de } \\
\text { Braden e Glasgow } \\
\text { para identificação de }\end{array}$ & 2008 & Estudo descritivo e exploratório & $\begin{array}{l}\text { Os resultados confirmaram que esses instrumentos podem } \\
\text { auxiliar o enfermeiro na identificação de pacientes em risco } \\
\text { para o planejamento da assistência }\end{array}$ \\
\hline
\end{tabular}


Research, Society and Development, v. 10, n. 17, e61101723945, 2021

(CC BY 4.0) | ISSN 2525-3409 | DOI: http://dx.doi.org/10.33448/rsd-v10i17.23945

\begin{tabular}{|c|c|c|c|}
\hline $\begin{array}{c}\text { risco para úlceras de } \\
\text { pressão em pacientes } \\
\text { internados em centro } \\
\text { de terapia intensiva }\end{array}$ & & & \\
\hline $\begin{array}{l}\text { Prevenção de } \\
\text { tratamento de lesão } \\
\text { por pressão }\end{array}$ & 2020 & Departamento de informática do sistema único de saúde & ------- \\
\hline $\begin{array}{c}\text { Prevenção e } \\
\text { terapêutica no } \\
\text { tratamento de lesões } \\
\text { por pressão: uma } \\
\text { revisão integrativa }\end{array}$ & 2021 & $\begin{array}{l}\text { Trata-se de uma revisão integrativa da literatura } \\
\text { realizada nas bases de dados: LILACS, MEDLINE, } \\
\text { BVS, BDENF. Foram selecionados } 16 \text { artigos no período } \\
\text { de } 2010 \text { a } 2020 \text { em português, inglês e espanhol que } \\
\text { tratavam sobre prevenção e terapêutica de lesão por } \\
\text { pressão. }\end{array}$ & $\begin{array}{l}\text { Apesar dos avanços tecnológicos são necessários maiores } \\
\text { investimentos por partes dos gestores dos serviços de saúde e } \\
\text { um maior comprometimento dos membros da equipe } \\
\text { multiprofissional para utilizarem os recursos já conhecidos e } \\
\text { eficazes para a prevenção e tratamento destas lesões a fim de } \\
\text { preservar e/ou restabelecer a saúde do indivíduo que precisa } \\
\text { de cuidados. }\end{array}$ \\
\hline $\begin{array}{l}\text { Ozionioterapia em } \\
\text { lesão por pressão } \\
\text { como alternativa de } \\
\text { assistência em } \\
\text { enfermagem }\end{array}$ & 2021 & $\begin{array}{l}\text { Trata-se de uma revisão bibliográfica baseada em artigos } \\
\text { nacionais e internacionais, com pesquisa em base } \\
\text { eletrônica de dados científicos tais como: Biblioteca } \\
\text { Virtual em Saúde (BVS), Scielo e Google Acadêmico, } \\
\text { como referências técnico-científicas brasileiras em } \\
\text { enfermagem }\end{array}$ & $\begin{array}{l}\text { E os resultados encontrados indicam a importância do uso } \\
\text { dessa prática como opção de assistência com a obtenção de } \\
\text { excelentes taxas de melhora }\end{array}$ \\
\hline $\begin{array}{l}\text { Terapia por pressão } \\
\text { negativa no tratamento } \\
\text { de feridas complexas }\end{array}$ & 2017 & $\begin{array}{l}\text { Foi realizada revisão na base de dados Pubmed,medline } \\
\text { em artigos publicados de } 1997 \text { a 2016, e selecionados os } \\
\text { mais relevantes. }\end{array}$ & $\begin{array}{l}\text { As principais indicações da TPN são as feridas complexas } \\
\text { como úlceras por pressão, feridas traumáticas, deiscências de } \\
\text { ferida operatória, queimaduras, feridas necrotizantes, úlceras } \\
\text { venosas, feridas diabéticas, os enxertos de pele, o abdome } \\
\text { aberto, na prevenção de complicações em incisões fechadas e } \\
\text { na associação com instilação de soluções em feridas } \\
\text { infectadas }\end{array}$ \\
\hline
\end{tabular}

Fonte: Autores.

Após a aplicação dos critérios de inclusão e exclusão, foi realizada uma leitura flutuante dos artigos que potencialmente atendiam esta revisão. Dos 59 artigos inicialmente selecionados, vinte foram encontrados no Scielo, quinze no Portal BVS, três na PubMed, 10 LILACS e 11 no Capes Periódicos. Mediante essa busca, foi realizada uma leitura interpretativa dos artigos a fim de buscar estudos que tivessem aderência à temática com o intuito de atender ao objetivo proposto neste estudo. Após a leitura dos títulos e resumos desses artigos, foram eleitas 36 publicações para leitura na íntegra, sendo selecionados 20 artigos para o estudo dessa pesquisa.

Quadro 2. Escala de Braden para prevenção das lesões por pressão.

\section{ESCORES}

Variáveis

Percepção sensorial

Umidade

Mobilidade

Nutrição

Fricção e cisalhamento
1

2

\begin{tabular}{|l|l|l|} 
Muito limitado & $\begin{array}{l}\text { Pouco limitado } \\
\text { Ouito molhado }\end{array}$ & $\begin{array}{l}\text { Nenhuma limitação } \\
\text { molhado }\end{array}$ \\
Confinado a cadeira & $\begin{array}{l}\text { Caminha } \\
\text { ocasionalmente }\end{array}$ & Raramente molhado \\
Muito limitado & Pouco limitado & Sem limitações \\
Inadequada & Adequado & Excelente \\
Problema potencial & Sem problema & ----------- \\
\hline
\end{tabular}

Fonte: DataSUS. 
A utilização de escalas de predição, como a Escala de Braden (EB), é comumente usada como parte de instrumentos metodológicos e diretrizes/protocolos clínicos para a prevenção de LP (Debon et al., 2018; Vieira et al., 2018; Alves et al., 2016). Assim, a escala de braden subsidia a avaliação clínica realizada pelo enfermeiro, bem como norteia o julgamento clínico e a tomada de decisões, auxiliando assim na construção de um plano de cuidados individualizado para identificar e impedir que pacientes desenvolvam LP, norteando assim a escolha de medidas preventivas apropriadas e efetivas para cada paciente (Alves et al., 2016; Debon et al., 2018).

Quadro 3. Escala de Braden.

BRADEN
• Escore: menor que $12=$ risco alto/muito alto
• Escore 13 e $14=$ risco moderado
- Escore 15 a $18=$ risco leve
- Escore $=$ maior que $19=$ sem risco

Fonte: Datas SUS.

Neste quadro identifica a avaliação do grau de risco de desenvolvimento de lesão por pressão. o escore da escala de Braden identifica a classificação dos pacientes quanto ao risco para LPP, portanto é obtida pela aplicação da escala de Braden em adultos mediante os escores.

O estudo apresenta evidências para o tratamento de pacientes que desenvolvem LP. Todos os estudos incluídos na revisão são baseados em ensaios controlados randomizados e, portanto, todos apresentam nível de evidência científicas quer subsidiam, fortalecem e tornam o cuidado mais efetivo e seguro. Por meio dos estudos randomizados controlados, é possível controlar as variáveis relacionadas às características dos pacientes, comorbidades, uso de fármacos, entre outros.

A escala de Braden fornece seis parâmetros para avaliação, pelas suas subescalas: 1- percepção sensorial; 2- umidade; 3- atividade; 4- mobilidade; 5- nutrição; 6- fricção e cisalhamento. Cada subescala tem pontuação que varia entre 1 e 4, com exceção do domínio fricção e cisalhamento. A somatória total fica entre os valores 6 e 23 , Escore igual ou menor que 16 denota que o paciente adulto tem risco para o desenvolvimento de LPP, entretanto, na presença de outros fatores como idade maior que 65 anos, febre, baixa ingestão de proteína, pressão diastólica menor que $60 \mathrm{mmHg}$ e/ou instabilidade hemodinâmica os pacientes com escores 17 e 18 também são considerados como pacientes de risco, Para pacientes críticos, o risco pela escala de Braden é estabelecido como sendo: risco baixo - escores entre 15 e 18; risco moderado - escores entre 13 e 14; risco elevado - escores entre 10 e 12; risco muito elevado - escores de 9 ou menor.

A pele é formada por duas camadas principais, que são a epiderme e a derme, além do tecido subcutâneo, que serve para uni-la aos demais tecidos. A epiderme é formada por uma camada de células epiteliais sobrepostas e não contém vasos sanguíneos, mas tem terminações nervosas. A derme é uma camada de tecido conjuntivo denso, constituídas

por fibras colágenas e elásticas entrelaçadas, sobre a qual se apoia a epiderme; que dá suporte a vasos, nervos, glândulas sebáceas, sudoríparas e folículos pilosos. O tecido subcutâneo situa-se entre a derme e a fáscia muscular. E é formado principalmente por um tecido conjuntivo frouxo (colágeno e elastina) e tecido adiposo. (Moissi-Eichnger et al., 2017)

A lesão por pressão é uma alteração na pele, normalmente próxima a regiões de proeminências ósseas, onde ocorre uma pressão que não foi atenuada. Também se denomina como LPP, toda injúria que surge próxima à dispositivos médicos, na qual também houve uma pressão na pele ou mucosa que não foi aliviada. (Ferreira., Renovato et al., 2020)

A lesão, que se apresenta em pele íntegra ou como úlcera aberta, pode ser dolorosa e ocorre como resultado da pressão intensa ou prolongada em combinação com o cisalhamento. Para classificar a gravidade das LLP, a NPUAU (national pressure 
ulcer advisory panel) propôs um sistema que considera como estágios/graus de desenvolvimento das mesmas, o estágio 1 referese a pele íntegra com eritema que não embranquece; estágio 2, perda da pele em sua espessura parcial com exposição da derme; estágio 3, perda da pele em sua espessura total; e estágio 4, é a perda da pele em sua espessura total e perda tissular. As lesões por pressão ainda podem ser classificadas como lesão por pressão não classificável e lesão por pressão tissular profunda. (Guideline.2014)

Durante o último encontro do NPUAP, (national pressure ulcer advisory panel) ocorrido em abril de 2016, outras definições de LPP foram adicionadas: lesão por pressão relacionados a dispositivo médico e lesão por pressão em membrana mucosa. A NPUAP descreve a lesão por pressão relacionada a dispositivo médico por sua etiologia que resulta do uso de dispositivos criados e aplicados para fins e diagnósticos terapêuticos. A lesão geralmente apresenta o formato do dispositivo que a originou. (NPUAP, 2016).

O outro aspecto que não pode ser esquecido é o estadiamento das lesões, sendo esta a irreversibilidade dos estágios. Portanto uma ferida com envolvimento subcutâneo será preenchida por tecido de granulação e se contrai, ela não é preenchida pelo mesmo tecido que havia originalmente. Consequentemente uma lesão classificada como estágio 4 não poderá voltar a ser estágio 3,2 e 1, pois já não existem mais, os marcadores tissulares definidores. Utiliza-se então o estágio original (último a ser classificado) e a descrição da profundidade atual da lesão (Rosa., 2016).

A prevenção de LPP representa um cuidado indispensável, visto que evitar que a lesão se desenvolva desprende menos trabalho da equipe comparado ao tempo e aos custos necessários para lidar com as consequências do surgimento das LPP. A carência em ações preventivas culmina com o desenvolvimento das lesões por pressão e a partir dessa realidade, torna-se necessário a implementação de um tratamento imediato e eficaz que pode minimizar os efeitos nocivos da lesão, tornando mais rápida a recuperação do paciente. (Guideline, 2019).

Devido ao impacto do desenvolvimento das úlceras por pressão na saúde do indivíduo, é fundamental que a avaliação do risco seja realizada de modo a planejar e iniciar intervenções preventivas personalizadas. (Guideline, 2014)

O profissional de enfermagem deve incluir medidas preventivas para que obtenha bons resultados a partir de uma avaliação completa da pele, e/ou as avaliações de risco para verificar eventuais alterações em uma pele intacta. Documentar todas as avaliações do risco. Desenvolver e implementar um plano de prevenção baseado no risco para os indivíduos identificados como estando em risco de desenvolver úlceras por pressão. Considerar o impacto das limitações de mobilidade no risco das úlceras por pressão. Os indivíduos acamados ou confinados a cadeiras de rodas são geralmente descritos como tendo limitações de atividade. Uma redução da frequência de movimento ou da capacidade de um indivíduo se mover é normalmente considerada como uma limitação da mobilidade. Realizar uma avaliação completa do risco em indivíduos acamados e/ou confinados a cadeiras de rodas para orientar intervenções preventivas. É importante a avaliação da pele, diferenciar se o eritema da pele é branqueável ou não branqueável. Utilizar o dedo ou o método do disco para avaliar se a pele é branqueável ou não branqueável. método de pressão com os dedos - o dedo é pressionado sobre o eritema durante três segundos e o branqueamento avaliado após remoção do dedo; método do disco transparente - é utilizado um disco transparente para pressionar uniformemente uma área de eritema, podendo observar-se o branqueamento sob o disco durante a sua aplicação (Guideline, 2019).

Recomenda-se como medidas preventivas a mudança de decúbito, a cada 2 horas (quando não existe contraindicação) aplicação de curativo protetor não aderente, utilização de coxins, uso de hidratação, e uso de colchão pneumático, e o uso de ácidos graxos essenciais (AGE) e hidrocoloide para prevenção. Para limpeza é indicada solução fisiológica (0.9\%), aquecida, pois soluções frias reduzem o fluxo sanguíneo nas áreas lesionadas e retardam o processo cicatricial (Correia \& Santos; 2019). 
O tratamento das lesões necessita de uma intervenção, centrada em abordagem holística, norteando os profissionais da saúde a fundamentação da prática em evidências científicas. É necessário avaliar o tipo de tecido presente na lesão e a quantidade de exsudato presente a fim de direcionar a melhor conduta terapêutica.

O surgimento de novas tecnologias tem permitido o aumento da disponibilidade de produtos no mercado. Na área de dermatologia, tem se encontrado cada vez mais produtos para o tratamento das feridas. Que visam otimizar o processo de cicatrização das lesões e proporcionar os pacientes as melhores condições de tratamento e recuperação. Contudo, a grande variedade de materiais, da mesma maneira que possibilita uma melhor adequação e manejo dos diversos tipos de feridas, também demanda um conhecimento especializado na área. (Rodrigues et al.,2015).

A terapia por pressão negativa (TPN) é um tipo de tratamento ativo da ferida que promove sua cicatrização em ambiente úmido, por meio de uma pressão subatmosférica controlada e aplicada localmente. A TPN é composta por um material de interface (espuma ou gaze), por meio do qual a pressão subatmosférica é aplicada e o exsudato é removido. Esse material fica em contato com o leito da ferida com objetivo de cobrir toda sua extensão, incluindo túneis e cavidades. O material de interface é coberto por uma película adesiva transparente que oclui totalmente a ferida em relação ao meio externo. Em seguida, um tubo de sucção é conectado a esse sistema e ao reservatório de exsudato, que é adaptado a um dispositivo computadorizado. Esse dispositivo pode permitir a programação de parâmetros para fornecer uma pressão subatmosférica no leito da ferida, possui alarme sonoro que indica eventual vazamento de ar pelo curativo e pode indicar a necessidade de troca do reservatório.

Dentre os recursos para o tratamento, o uso de curativos com diferentes tipos de coberturas é considerado a terapia convencional. Eles beneficiam o processo de cicatrização a médio e longo prazo, dependendo do estadiamento, com base no grau de destruição tecidual das lesões e das condições do paciente (Bernardes; Meska; et al., 2019).

Agentes terapêuticos, como papaína nas concentrações de 8 a $10 \%$, hidrogel com alginato de cálcio e colagenase, são considerados tratamentos de escolha nos casos de LPP com presença de tecido desvitalizado (esfacelo, necrose, sem presença de exsudato). E para tecidos vitalizados, utiliza-se o agente terapêutico hidrogel, papaína 2\%, hidrogel com alginato, ácidos graxos essenciais e petrolato. Quanto ao tecido de epitelização, utiliza-se ácidos graxos essenciais, filme transparente e hidrocoloide extra fino, que são utilizados para reduzir as forças de friç̧ão e proteger áreas neoepitelizadas. (Carvalho; Salomé; Ferreira; 2017).

Durante o tratamento, é imprescindível que o profissional da enfermagem reavalie o paciente, a lesão por pressão e o plano de cuidados definido. Caso a lesão não mostre os sinais de cicatrização esperados, apesar dos cuidados locais adequadas a lesão, espera-se alguns sinais de cicatrização da lesão por pressão no prazo de duas semanas, assim ajustando as expectativas de cicatrização na presença de múltiplos fatores que dificultem essa cicatrização, senão houver progressos na cicatrização da lesão, no prazo de duas semanas. O paciente, a lesão por pressão e os planos de cuidados devem ser reavaliados. Assim informando o paciente sobre essa respectiva significativa sobre: o processo ou deterioração e os sinais e sintomas, que serão orientados pelos profissionais da saúde (Guideline; 2016).

Cada estágio da lesão por pressão requer a utilização de um cuidado e tratamento especifico. No estágio 1, utiliza-se a aplicação de TCM (triglicerídeos de cadeia média) ou AGE (ácidos graxos essenciais), no estágio 2 deve ser feita uma limpeza da ferida com jatos de soro fisiológico (0.9\%) morno utilizar hidrocoloide extrafino durante o período de 3 a 7 dias, (quando tem pequena quantidade de exsudato) ou óxido de zinco ou AGE ou hidrogel associado com gaze não aderente, (quando tem presença de grande quantidade de exsudato). no estágio 3, deve-se fazer a limpeza da ferida com jato de soro fisiológico (0.9\%) morno, na ausência de necrose é usado hidrogel com AGE em toda a sua extensão, com a presença de necrose utiliza-se colagenase e/ou papaína $8 \%$ sobre a necrose. Para preencher a cavidade usa-se uma gaze não aderente, no estágio 4, na ausência de necrose utiliza-se o hidrogel ou AGE em toda a sua extensão, preenchendo a sua cavidade com gaze não aderente, e na presença de 
necrose coloca-se colagenase, ou papaína 8\% sobre a necrose preenchendo a sua cavidade com gaze não aderente. (Bortolettiet al., 2015).

Nesse contexto, a identificação dos fatores individuais de risco é útil para realização de uma Sistematização da Assistência de Enfermagem no cuidado e na terapêutica. A utilização de escalas de avaliação do risco de desenvolvimento de LPP é de grande valia para a enfermagem e proporciona um planejamento da assistência ao paciente hospitalizado de modo sistematizado, o que facilita os processos de diagnóstico, tratamento e prevenção dessas lesões(9).A Escala de Braden é um dos instrumentos que auxilia na detecção dos riscos de desenvolver LPP, além de possibilitar aos profissionais de enfermagem um melhor delineamento na elaboração das prescrições dos cuidados que deverão ser oferecidos a esses pacientes (Jansen et al., 2019).

\section{Conclusão}

Neste presente trabalho, conclui-se que as estratégias de prevenção dos fatores de risco causadores de lesão por pressão, são feitas a partir da inspeção da pele, mudança de decúbito, nutrição adequada, hidratação da pele, uso de óleos e pomadas, uso de colchões de ar e casca de ovo e coxins, adequando-se ao uso da escala de braden, para fazer o uso do tratamento a avaliação tem que ser adequada e criteriosa para definição do grau/estágio da lesão, e uso correto de pomadas e placas, que melhorem a determinada lesão. Que seja avaliada por um profissional adequado e que tenha conhecimento sobre as lesões para definir o melhor plano terapêutico, para prevenção e tratamento da lesão. Além disso, é importante a realização de novos estudos sobre o tema para ajudar na prevenção e tratamento de LPP, assim incentivando a busca de novas pesquisas, com intuito sempre de informar sobre as devidas atualizações do determinado assunto, trazendo benefícios para os leitores em busca de inovação, e da saúde em si.

\section{Referências}

Alessandra, M., Miranda, G. B. F., Meska, G. H. M., Bianchini, A., Bernardes, M. R., \& Júnior, P. A. G. (2017). Ensino de Prevenção e Tratamento de Lesão por Pressão Utilizando Simulação. www.scielo.br/j/ean/a/7D7LLFyVjVM7LGxwmym44FNf/?format=pdf\&lang-pt.

Alencar, G. S. A., Silva, N. M., Assis, E. V., Sousa, M. N. A., Pereira, J. L. F., Oliveira, W. B., \& Souza, E. F. (2018). Lesão Por Pressão Na Unidade de Terapia Intensiva: Incidência e Fatores de Riscos. Revista Enfermagem.

Abril2018/lesao_por_pressao_na_unidade_de_terapia_intensiva.pdf.

Bastos, M. D., Batista, L. D. J., Santos, A. A. J., Muniz, C. L. M., Muniz, C. L. M., Araujo, A. O. M. P., Rodrigues, S. I., Moura, C. C. A., Silva, M. V. K., \& Souto, L. T. (2021). prevenção e terapêutica no tratamento de lesão por pressão: uma revisão integrativa, revista eletrônica acervo saúde.13(7). https://doi.org/10.25248/REAS.e8091.2021.

Carvalho M. R. F., Salomé, G. M., \& Ferreira, L. M. (2017). Revista de enfermagem, UFPE.11(10), 4171-83.

Copyright National Pressure Úlcer Advisory Panel. (2016). NUAP, SOBEST. Associal Brasileira de Estomaterapia. https://sobest.com.br/wpcontent/uploads/2020/10/CONSENSO-NPUAP-2016_traducao-SOBEST-SOBENDE.pdf.

Correia, B. S. A., \& Santos, C. B. I. .2019). Lesão Por Pressão: Medidas Terapêuticas Utilizada por Profissionais de Enfermagem. Revista Brasileira de Ciências da Saúde. periodicos.ufpb.br/ojs2/index.php/rbcs/article/view/36793-p4/22325.

Departamento de informática do sistema único de saúde (DATASUS).

Favreto, L. J. F., Betiolli, L. S., Silva, B. F., \& Campa, A. (2017). O papel Do Enfermeiro Na Prevenção, Avaliação e Tratamento Das Lesões Por Pressão. Revista Gestão \& Saúde. www.herrero.com.br/files/revista/filea2aa9e889071e2802a49296ce895310b.pdf.

Fernandes, M. L., \& Carili, L. H. M. (2008). Uso da escala de Braden e de Glasgow para identificação do risco para úlceras por pressão em pacientes internados em centro de terapia intensiva. Revista Latino Americana de Enfermagem. 16(6). www.eerp.usp.br/rlae

GBSERH. (2018). Hospitais universitários federais, prevenção e tratamento de lesão por pressão, versão 1.0. www.2.ebserh.gov.br.

Guideline, (2016). Prevenção e tratamento de úlceras por pressão: guia de consulta rápida.

Guideline. (2019). Prevenção e tratamento de úlceras/lesões por pressão: guia de consulta rápida. 
Research, Society and Development, v. 10, n. 17, e61101723945, 2021

Jansen, S. C. R., Silva, A. B. K., \& Moura, S. E. M. (2020). Braden Scale in pressure ulcer risk assessment, revista brasileira de enfermagem.73(6), $2019-0413$. http://dx.doi.org/10.1590/0034-7167-2019-0413

Lima, R. N., Lima, R. N., Souza, O. C. J., Silvério S. T., Souza Filho, A. O. J. \& Santos-Nascimento T. (2021). Escala de braden: benefícios de sua aplicação na prevenção de lesão por pressão no âmbito domiciliar, Arquivos De Ciência Da Saúde Da Unipar. 25(2),95-103.

Lima, S. K. V. R., Coltro, S. P., \& Júnior, F. A. J. (2017). terapia por pressão negativa no tratamento de feridas complexas, Revista do Colégio Brasileiro de Cirurgiões. 44(1),081-093. https://www.scielo.br/j/rcbc/a/W6qy4BFN9DkdTRsGy6jrfkk/?format=pdf\&lang=pt.

Lamão, L. CL., Quintão, A. V., \& Nunes, R. C. (2016). Cuidados de Enfermagem na Prevenção de Lesão por Pressão. Revista Cientifica Interdisciplinar. www.multiploacessos.com/multaccess/index.php/multaccess/article/view/10/10.

Oliveira, G. S. M., Silva, R. K., Melo, B. S. C, \& Lemos, M. C. A. (2021). Ozionoterapia em lesão por pressão como alternativa em assistência de enfermagem, research, society and development. 10(3), 2525-3409.http://dx.doi.org/10.33448/rsd-v10i3.13777.

Galetto, S. G. S., Nascimento, P. R. E., Hermida, V. M. P., \& Malfussi, H. B. L. (2018). Lesão Por Pressão Relacionadas a Dispositivos Médicos: Revisão Integrativa Da Literatura. Revista Brasileira de Enfermagem. www.scielo.br/j/reben/a/BzhBLh3dBvdFKdHZWG4TLWG/?lang=pt.

Constante, R. A. S., \& Oliveira, C. V. (2018). Lesão Por Pressão: Uma Revisão de literatura. Revista Psicologia Saúde em Debate. psicodebate.dpgpsifm.com.br/index.php/periodico/article/view/V4N2A6/138.

Silva, A. R. D., Bezerra, G. M. S., Costa, P. J., Luz, A. B. H. M., Lopes A. C. V., \& Nogueira, T. L. (2017). curativos de lesões por pressão em pacientes críticos: análise de dados, UFPI, Teresina. Revista escola de enfermagem USP. 